\title{
Design and Realization of an IP-based In-car Network Architecture
}

\author{
Rainer Steffen, Richard Bogenberger, \\ Joachim Hillebrand, Wolfgang Hintermaier, \\ Andreas Winckler \\ BMW Group Research and Technology \\ Munich, Germany \\ \{firstname.lastname\}@bmw.de
}

\author{
Mehrnoush Rahmani \\ Technische Universitaet Muenchen \\ Media Technology Group \\ Munich, Germany \\ Mehrnoush.Rahmani@tum.de
}

\begin{abstract}
The requirements for in-car communication networks are constantly growing. A high amount of electronic control units with increasing communication demands have to be connected by a multitude of different automotive specific network technologies. All these facts lead to an increasing complexity and accordingly higher production cost.

To cope with these challenges there is a vision to do a radical step: The use of the Internet Protocol (IP) as an overall communication technology. This paper describes the design and realization of an IP-based in-car network architecture that replaces the existing in-car network technologies. We describe the benefits of the Internet Protocol as the enabler for future innovative in-car applications and define mechanisms to obtain the required quality of service and real-time behavior. Finally, we describe the implementation of an experimental vehicle with an integrated IP network as a proof of concept that covers all communication domains in the car.
\end{abstract}

\section{Keywords}

In-car network architecture, Internet Protocol.

\section{INTRODUCTION}

During the last years, the number of in-car communication networks has significantly grown due to the deployment of an increasing number of electronic control units (ECUs). Up to 70 different application specific ECUs are deployed in a premium car of the upper class that are connected by different bus systems like CAN, FlexRay, MOST, LIN etc. The network complexity will still grow in the future due to the increasing number of functions. Up to $90 \%$ of all innovations in a car are provided or assisted by modern electronic and software. Examples can be found in the

Permission to make digital or hard copies of all or part of this work for personal or classroom use is granted without fee provided that copies are not made or distributed for profit or commercial advantage and that copies bear this notice and the full citation on the first page. To copy otherwise, or republish, to post on servers or to redistribute to lists, requires prior specific permission and/or a fee.

ISVCS 2008, July 22 - 24, 2008, Dublin, Ireland.

Copyright 2008 ISBN \# 978-963-9799-27-1 area of infotainment and driver assistance systems as well as power train or safety systems.

To face the growing complexity and to comply with future demands, new concepts are needed. A radical vision that addresses the described challenges is the use of the Internet Protocol as a common basis for the in-car communication network.

The paper is organized as follows: section 2 motivates the vision of an all IP-based in-car network architecture and describes the main advantages. Section 3 shows how the Internet Protocol enables new applications and explains several use cases that benefit from the IP technology. Section 4 describes how quality of service and real-time communication can be assured to meet the automotive communication requirements. Section 5 deals with the implementation of an IP-based network into an experimental vehicle. Finally, section 6 concludes the paper and gives a brief outlook on our future work.

\section{VISION OF AN ALL IP-BASED IN-CAR NETWORK ARCHITECTURE}

The Internet Protocol can be seen as an abstraction layer between the applications and physical network technologies. By using IP, the in-car communication network speaks a common and universal language. The applications can utilize a standardized communication interface independent of the physical network. That means that the network technology (e.g. electrical Ethernet, optical Ethernet, WLAN, Bluetooth, UWB, etc.) can be replaced without the need of changing the application software. This is in principle not the case for current communication systems in the vehicle. Also heterogeneous networks, consisting of different physical transmission technologies, can easily be build by using the Internet Protocol (figure 1). They all speak the same 'language', namely the Internet Protocol.

If the data rate requirements increase from a vehicle generation to the next one so that the current network technology does not suffice any more, it can easily be replaced with a faster one without the need to change the application software. An example is the migration from Fast Ethernet (100 MBit/s) to Gigabit Ethernet in the car's communication backbone. 


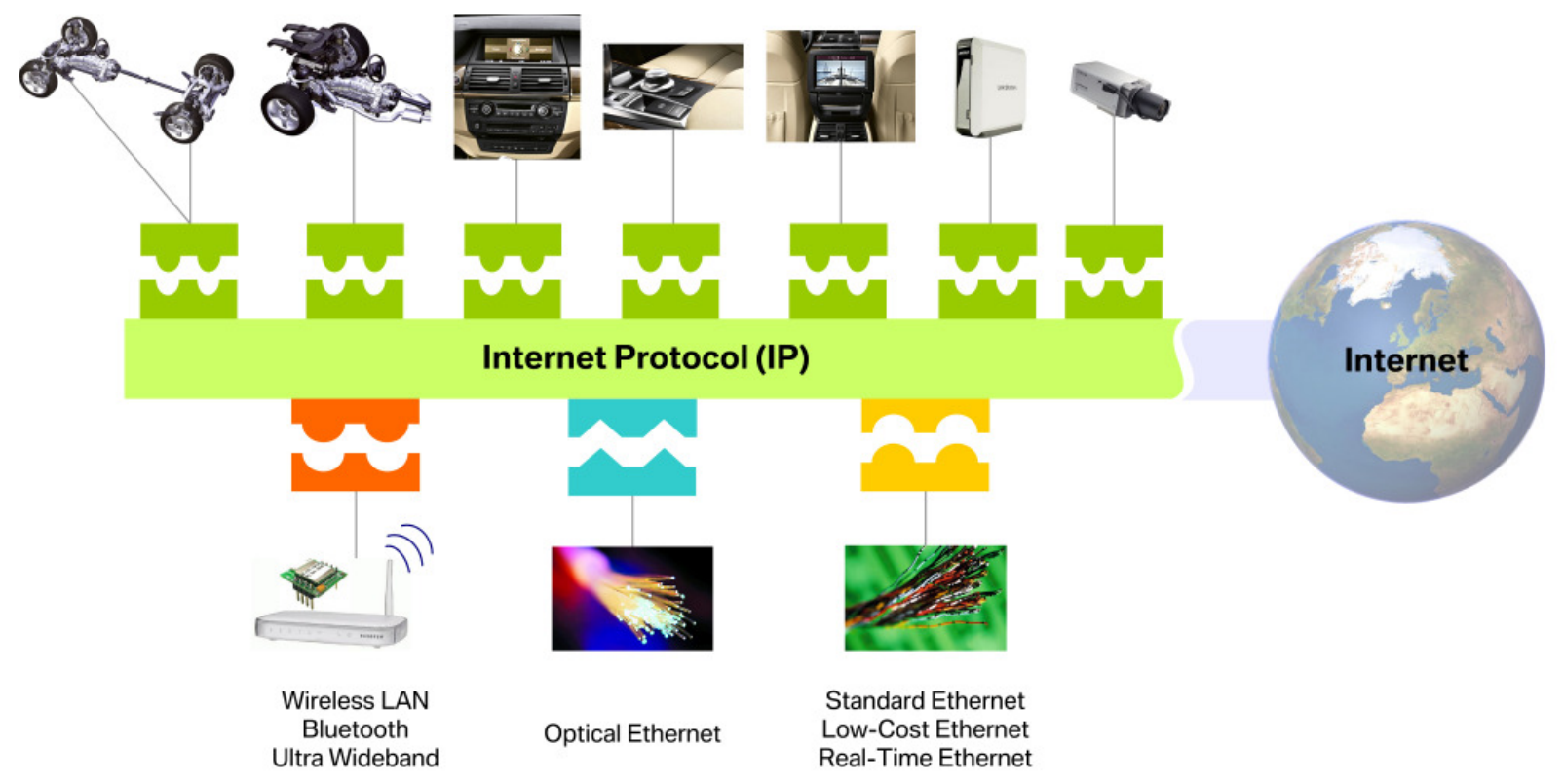

Figure 1: The Internet Protocol offers a common interface between the applications and the different physical network technologies

The described flexibility is emphasized by a modular and standardized software framework which is provided by the ITworld and that is also applicable for the restrictive resource requirements in the automotive world. This modular software framework provides different implementations of the TCP/IP protocol suite with all related protocols. Examples are Universal Plug and Play (UPnP) for controlling consumer electronic devices, the Real-Time Transport Protocol (RTP) for streaming audio and video data or the Simple Network Management Protocol (SNMP) for network management.

The vision of an all-IP in-car communication network entails further advantages regarding the design of the network architecture. By the introduction of IP it would be possible to resign complex gateways since there is no need to convert data and frame formats between completely different network technologies like MOST, CAN, FlexRay or LIN. Thus the gateway is reduced to a simple router of IP packets without the need of processing and converting the data. All this leads to a remarkable complexity reduction in the overall network architecture and to a simplification of the system design and partitioning.

IP has advantages also in terms of the development process. Comparing the different development tools and measurement equipment available for the IP technology with those available for the automotive domain, it becomes clear that the IP technology offers a much wider range with a lower cost. A very prominent example is the open source protocol analyzer 'wireshark' which is widely used in the internet area and which is available free of charge.

\section{IP AS AN ENABLER FOR NEW APPLICATIONS}

The Internet Protocol offers a variety of new applications that can easily be integrated into the car due to the standardized interfaces and the modular building blocks. The next sub-sections show a selection of applications where IP is the enabling technology with a multitude of benefits.

\subsection{The Car as a Part of the Private Communication Network}

Consumer electronic is a growing part of our daily life that is also entering the automotive market. The demand for using mobile equipments like MP3 players, mobile phones, or PDAs inside the car is increasing rapidly. The goal is to have a solution that allows the connection of mobile devices to the car in an intelligent way. One of the challenges is that the product life cycle of consumer electronic devices is much shorter than that of the automotive industry which leads to high integration and changing effort in the cars.

The IP technology and its related protocols and services offer a solution with which the short life consumer electronic devices can be integrated into the cars by long life standardized interfaces.

One example for such an interface is Universal Plug and Play (UPnP) [1] that is based on IP communication. UPnP can be used to control devices such as audio player, video devices, router, printer, control applications etc. in a vendor unspecific manner. The interface to the services is described with standardized device profiles in XML and allows vendor independent compatibility.

The UPnP device architecture defines the UPnP AV device profile that is particularly used to connect and control entertainment devices over IP. UPnP AV allows the control of 
different media formats like audio, video and images. The standard MMI (e.g. head-unit or the rear seat entertainment system) of the car can be used to control and display UPnP services.

\subsection{Internet Services}

By using the Internet Protocol, the car can easily be connected to the public internet for example by using GPRS, UMTS, WiMAX or other access technologies. This allows seamless access to a multitude of different services like voice over IP, video conferencing services, internet TV or IP-radio. With the IP technology new applications can fast and easily be built by using already existing software components from the IT-world. An example is a video based assistance system with which the driver can communicate over a video link with a personal service agent. Over this video link additional useful information can be transmitted like the function of a control element that the service agent can explain.

As the number of internet services is continuously growing there will be a huge amount of possible application and transmission scenarios. Considering Web 2.0 applications one can imagine which potential is behind the IP technology.

By opening the car to the internet, particular attention has to be paid to security aspects. The requirements concerning the security are growing with the increasing interconnection between the car and the outside world. The car has to be protected against unauthorized access and manipulation. But again, already available concepts from the IT-world offer a variety of different solutions that can be used. Firewalls, proxies, IPSec or VPNs are well known approaches that can be used to build an adequate security architecture for the car.

\subsection{Digital Camera Systems}

In addition to the audio- and video-based consumer electronic devices, also the number of camera systems and video transmitting sensors is growing mainly in the field of driver assistance services. Video-based driver assistance applications require high transmission capacities in order to send large video frames within short time intervals (see section 4.1 for detailed requirements).

Current automotive specific network systems have already reached their capacity bounds and are not easily extensible for future video applications. Up to now, video transmission is done by systems like analogue FBAS or digital low voltage differential signaling (LVDS). These point-to-point video connections are installed in addition to the already existing in-car networks which lead to an increased overall complexity and production cost.

A consistent network architecture can also be achieved for realtime in-car video communication by using IP over fast network technologies such as Ethernet. The currently used analogue camera systems can be replaced by digital one-chip cameras. These one-chip cameras are produced in large amounts for the consumer electronic market (e.g. mobile phone cameras) and have a very low price compared to the conventional in-car video technology.

In order to transmit several video streams over one Fast Ethernet link, video compression has to be applied at the sources. Our analysis has shown that the application of compression algorithms such as Motion JPEG 2000, MPEG-4/Part II, H.264 (MPEG-
4/AVC) [2] do not violate the quality requirements of in-car video applications, but reduces the capacity requirements as desired.

The introduction of IP-cameras which apply compression techniques like H.264 entails a huge technical and cost potential. By using these cameras it is possible to distribute all image data taken by the cameras to every place within the whole IP network. As opposed to the current situation, it is possible to reuse same cameras for different applications which leads to a significant cost and a topology complexity reduction. An example for reusing a camera is the top view camera system which can also be used for detecting time to lane crossing.

Thanks to the IP technology, scalability in terms of the amount of integrated cameras will be no problem anymore. In contrast to analogue video switches a conventional IP-switch is less cost intensive and provides much more flexibility in terms of topology diversity.

\section{QUALITY OF SERVICE PROVISIONING AND REAL-TIME COMMUNICATION}

The current automotive in-car networks are built for specific application fields. For example FlexRay was specially built for real-time communication of control data or MOST was build for entertainment services. All these specific networks fulfill a particular set of requirements like real-time capability or quality of service. By using IP as a convergence layer, the network has to be able to fulfill the requirements of all the different application areas. In order to define QoS mechanisms it is necessary to first determine the QoS requirements of the future network.

\subsection{In-car Traffic Categories}

We can classify the in-car traffic into the following traffic categories based on their QoS requirements [3]: Real-time control data, real-time audio and video data, multimedia data, best effort data.

\section{Category A: Real-time control data}

The requirements for the transmission of real-time control data depend on the particular ECU and its specific set of data that has to be transmitted. It is not useful to define common requirements for all kinds of ECUs but rather define worst case conditions that might be necessary to cover real-time needs of the current and future in-car communication systems.

Currently the strongest requirements concerning the end-to-end delay between two ECUs is $2.5 \mathrm{~ms}$. Most of the ECUs have minor requirements of mostly $\geq 10 \mathrm{~ms}$. A future IP based in-car communication system needs mechanisms to guarantee at least these requirements but must also be flexible enough to achieve lower end-to-end delays for future applications.

The basic mechanisms to guarantee the real-time behavior are described in section 4.3 . 


\section{Category B: Real-time audio and video data}

This traffic category contains all time critical multimedia data which are:

- Video data from camera systems or video transmitting sensor systems of driver assistance services.

- Interactive video applications such as video conferencing.

- Interactive audio applications such as VoIP.

We assume to have a maximum of 7 cameras within the car that stream compressed video data throughout the entire in-car network. System requirements for the video streams are given as follows:

- Frame rate: 30 frames/sec.

- Frame resolution: at least VGA $(640 \times 480$ pixels).

- Transmission rate: up to $7 \mathrm{Mbit} / \mathrm{sec}$. (average data rate for camera video streams)

- End-to-end delay: max. $45 \mathrm{~ms}$. When post processing is applied, the transmission time excluding the video processing time should be at most $33 \mathrm{~ms}$.

- Packet loss for an adequate video quality: $\leq 0.1 \%$ (depends on the applied video codec).

MPEG-coded videos consist of 3 frame types, that are I-, P- and $\mathrm{B}$-frames. However, the presence of B-frames imposes extra delay to the coding and decoding processes due to the fact that $\mathrm{B}$-frames make use of forward and backward prediction [2]. For this reason, we apply the MPEG-4 compression without B-frame coding. Our analyses based on software implemented video codecs have shown that the compression and decompression of video streams introduces a total delay of 10 to $20 \mathrm{~ms}$. This time can considerably be reduced by application of hardware based codecs that are assumed to be applied soon within the car.

\section{Category C: Multimedia data}

This traffic category contains data from in-car multimedia systems that transmit (compressed) audio and video data for entertainment of the car occupants.

These applications have strong requirements concerning the delay jitter. Usually buffers are applied at the receiver side in order to compensate the transmission jitter. However, to realize a low userperceived end-to-end delay either no buffer or a very small buffer should be applied at the receiver. The required transmission capacity is high, because of the large amount of data. These applications usually apply the PAL or NTSC format. But for the future, the high definition (HD) format has also to be considered. The QoS requirements of in-car multimedia applications are listed in the following:

Video data (e.g. DVD, TV)

- Transmission rate: 4-8 Mbit/sec (PAL), $27 \mathrm{Mbit} / \mathrm{sec}$ (HD).

- Frame rate: 25 frames/sec (PAL), 50 frames/sec (HD).

- Frame resolution: 720×576 (PAL), 1920×1080 (HD).

- End-to-end delay: max. $100 \mathrm{~ms}$ [4].

- Packet loss for an adequate video quality: $\leq 0.1 \%$.
Audio data (e.g. CD, MP3)

- Transmission rate: $128 \mathrm{kbit} / \mathrm{sec}$ (MP3), 1.4 Mbit/sec (Audio CD).

- Sampling frequency: $44.1 \mathrm{kHz}(\mathrm{CD}, \mathrm{MP} 3)$.

- End-to-end delay: max. $100 \mathrm{~ms}$ [4].

- Packet loss for an adequate audio quality: $\leq 0.1 \%$.

\section{Category D: Best effort data}

Best effort communication is characterized by the fact that it has no QoS requirements for the underlying network. The communication system ensures that packets are sent as soon as free resources are available. There is no guarantee for packet loss and delay times. This traffic category for example includes diagnosis, internet services and download of data.

\subsection{Priority Assignment - a Lightweight but Stable QoS Solution}

Due to its wide availability and the low cost, Ethernet is one preferred physical network technology. However, some quality of service mechanisms have to be applied to it in order to achieve the desired behavior for time constraint audio and video applications. In the following context, we assume to have a switched fullduplex Ethernet in order send and receive data at the same time without any collisions.

It is obvious that in switched full-duplex Ethernet networks, QoS should be provided and assured not only in the switches but also in all devices connected to the network.

In order to differentiate between the in-car traffic categories with different QoS requirements, we proposed the concept of static priority assignment in [5]. Since the in-car applications and their QoS requirements are known a priori, we can configure the network statically before the start-up. To do that, we apply the concept of IP Diffserv [6] in combination with the IEEE 802.1p [7] standard. This allows us to define eight different priority classes that differentiate the respective traffic categories with their QoS requirements.

The configuration of QoS requirements is done in a QoS API that is able to assign priority levels to the data packets (figure 2). This QoS API is an extension to the standard protocol stack and works in parallel to the communication APIs (e.g. socket interfaces).

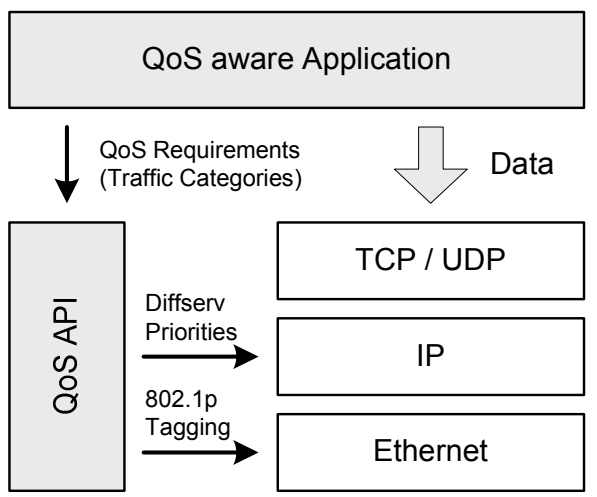

+ optional traffic regulation

Figure 2: QoS configuration through a QoS API 
The QoS API is either directly controlled by the application if it is a QoS aware application or it is controlled by an external script when it is a legacy application without any QoS support.

Obviously, this QoS concept requires an appropriate network dimensioning. Since the in-car communication demands are known a priori, the network setup can be calculated in advance. Mathematical analysis methods and simulations can be used to find the optimal settings of the network (topology, link speed, buffer sizes, priority assignments, etc.).

Experimental prototypes as well as simulations show that the described system can fully meet the QoS requirements described in section 4.1 for multimedia and infotainment systems. By the use of IP in combination with Ethernet, a high quality and a stable behavior can be achieved.

\subsection{Real-Time Communication with IP}

The described scenarios and applications show that the Internet Protocol can be used efficiently in the infotainment and multimedia domain. Additionally IP also has a high potential for applications in the real-time domain that is up to now exclusively served by automotive-specific bus systems like CAN or FlexRay. By bringing IP into the real-time domain, both multimedia information and time critical control messages have to be transmitted over the same IP network.

As a consequence, the IP-based network has to guarantee the communication delay with hard bounds. Such hard real-time mechanisms are neither implemented in the Internet Protocol nor in the standard Ethernet. Nevertheless, by using appropriate mechanisms like adequate network dimensioning in combination with traffic shaping mechanisms, real-time constraints can be guaranteed. This could be done without violating the Ethernet or Internet Protocol standard. The real-time behavior has been verified analytically, in real test and evaluation systems as well as in the experimental vehicle (section 5).

To synchronize the ECUs, the Precision Time Protocol (PTP; IEEE 1588) can be used. This protocol is well known from the automation industry and gives all networked devices a common time basis with a very high accuracy. This enables unique time stamps within the in-car network that can be used for data acquisition or to send data at discrete times.

Similar approaches for hard real-time communication can also be found in the aviation industry. The "Avionics Full Duplex Switched Ethernet" (AFDX) [8] is used by Airbus for time- and safety-critical data communication within the new Airbus A380. Boeing is using the Common Data Network (CDN) in its new Dreamliner (Boeing 787) that is based on a similar technology as AFDX.

Both, our own evaluations and experiments as well as the experiences from the non-automotive industries show that the Internet Protocol in combination with Ethernet is able to even handle the hard real-time traffic.

\section{REALISATION OF AN IP-BASED IN-CAR NETWORK}

In order to show that the IP technology is not only appropriate in theory and in the laboratories, but also in real cars, an experimental vehicle has been built. An IP-based network was implemented into a BMW 530d that covers the following application domains: infotainment/multimedia, internet applications and real-time communication of control data. The network architecture of the prototype car is shown in figure 3.

A customized IP-based head-unit software has been built and used as the basis for the application scenarios. The head-unit software

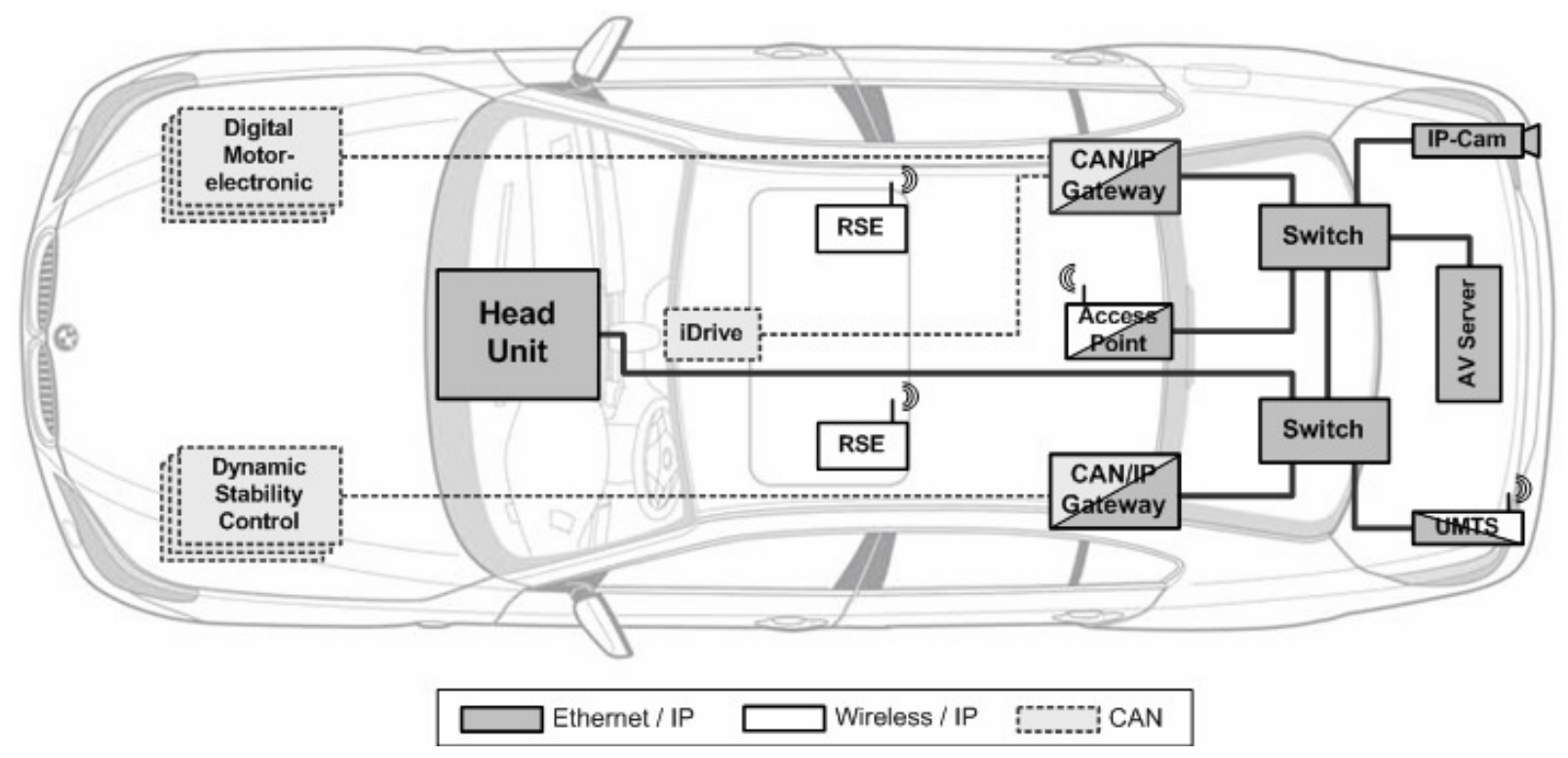

Figure 3: Configuration of the experimental vehicle. Multimedia as well as real-time data is transported over the same network. 
allows UPnP-based access to the car's internal media server for audio as well as video streaming applications. UPnP enabled consumer electronic devices can be connected via WLAN to the in-car network and have full access to the in-car media server. In addition to that, they can also transmit their content to the in-car network.

In order to demonstrate the flexibility of the system a rear seat entertainment system was built. The rear-seat entertainment system consists of two Tablet PCs that are connected via WLAN to the in-car network. The Tablet PCs are running with the same software as the head-unit and have exactly the same functions. This is due to the fact, that every data can be transmitted to every device within the IP-based in-car network without any gateway or data transformation. For example, this allows to watch a DVD video on one of the Tablet PCs while on the other one the IPbased rear view camera video is running.

The experimental vehicle is connected to the outer world either by UMTS or by WLAN. Thus, the car has a full internet access. This internet connection is a basis for applications like IP-TV, internet radio or a video based assistance service as described in section 3.2. Security mechanisms that protect the in-car network from the public internet are already implemented into the experimental vehicle.

The access to the car's internal legacy bus systems (e.g. CAN bus) as well as the real-time communication of control data have been implemented. To demonstrate the real-time behavior of the IP network, the CAN connection between the dynamic stability control system (DSC) and the digital motor electronic was disconnected. These CAN messages were then fed into the IP network using a CAN/IP-gateway (figure 3). Static measurements as well as test runs with the car showed that the IP communication works error free (figure 4). Even if several multimedia streams were sent over the same IP network no errors occurred and the timing requirements could always be met.

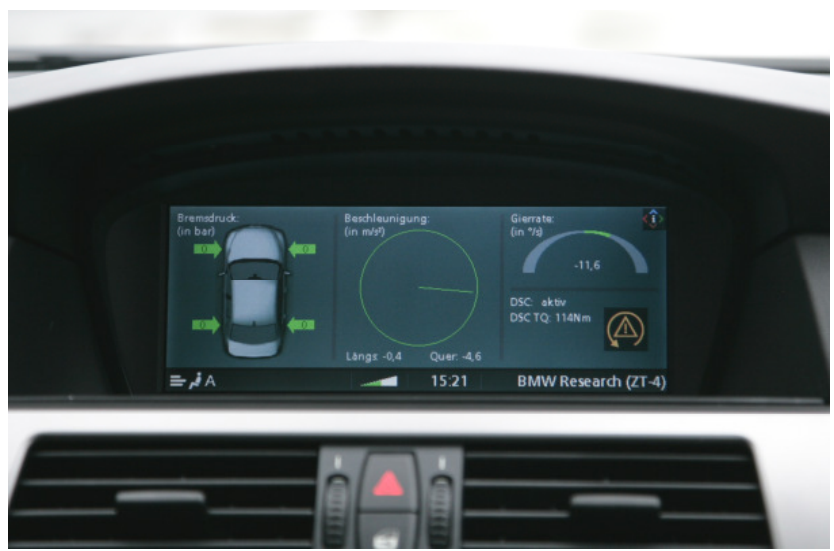

Figure 4: The Dynamic Stability Control (DSC) system sends control data in real-time over IP. The head-unit shows selected parameters of that control system.
All these examples have shown that the concept of an IP based incar network is practically working. Every communication device within the car can potentially communicate over IP. This solution would bring many advantages but also lead to a radical redesign of current in-car architectures. Figure 5 shows the trunk of the experimental vehicle (BMW 530d) with some of the network devices.

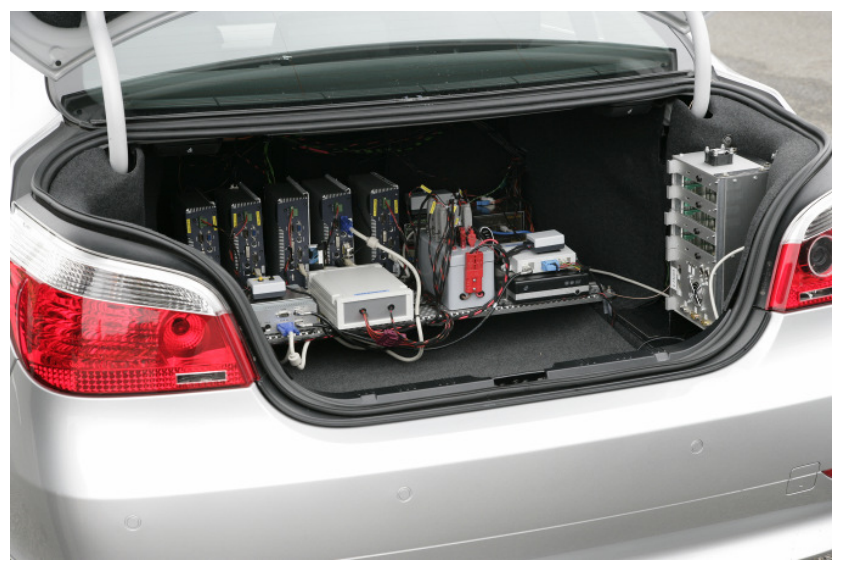

Figure 5: Research prototype of the IP in-car network

\section{CONCLUSION AND OUTLOOK}

This paper described a vision of an all IP-based in-car network and showed that the Internet Protocol is capable to fulfill all requirements of the current and future in-car applications.

It is obvious that it might not be reasonable to provide every ECU with a full IP stack and connect it to a high speed Ethernet network. For example current LIN-based systems are resource optimized low cost systems where the benefits of IP communication can not be justified considering its higher price.

In other application fields such as multimedia, infotainment and driver assistance application but also in the field of high performance real-time communication, IP is a serious alternative technology with many advantages and benefits.

\subsection{Benefits of an IP based In-car Communication Network}

Benefits in communication

- Common communication API.

- Common addressing scheme.

- Inter-domain communication without gateways.

- Standardized protocols (SNMP, PTP, NTP, etc.)

- Communication is independent from the physical network technology.

- Due to a common data format, there is no need for application- und bus-specific conversion of messages in a gateway.

$\Rightarrow$ Simplification of the in-car communication. 
Benefits in the development process

- Variety of already existing and cost-effective development tools available.

- Access to a plurality of different software modules.

- Existence of a large development community and know-how.

- New technologies (e.g. multimedia application, web 2.0) are IP based and can fast and easily be integrated into the car.

$\Rightarrow$ Faster and easier development with higher quality.

Benefits for the applications

- Applications can be developed independently from the physical network technology.

- Reuse of already existing applications.

- Faster and easier flashing, diagnosis and test.

- Easier shifting of function between different ECUs.

$\Rightarrow$ Simplification of application development and faster integration and test.

Further advantages

- Simplified connection of the car to corporate networks or backend systems (e.g. for development, production, maintenance, diagnosis, etc.)

- Internet Protocol is a standardized communication protocol and allows an enduring and sustainable utilization due to its extensive deployment in different industrial areas.

Not only car manufacturers and component suppliers but also the clients have a high benefit from the IP technology. It offers a fast integration of new applications and an easier communication with IP-based services.

\subsection{Future Work and Open Issues}

The feasibility of an all IP-based communication system has been shown by theoretical analysis but also by the integration of the IP technology into a experimental vehicle. But there are still some open issues that have to be considered in the future work such as:

- Electromagnetic compatibility (EMC).

- Automotive requirements for an IP aware hardware.

- Migration scenarios from legacy in-car communication systems to an IP-based network and the effects on the car's and ECU's architecture.

- IP for safety critical applications (e.g. redundancy)

- Harmonization / standardization of the IP technology for incar networks

\section{REFERENCES}

[1] M. Jeronimo, J. Weast, "UPnP Design by Example - A Software Designer's Duide to Universal Plug and Play", Intel Press, 2003.

[2] T. Wiegand, G. J. Sullivan, G. Bjontegaard, A. Luthra, "Overview of the H.264/AVC Video Coding Standard", IEEE Transactions on Circuits and Systems for Video Technology, Vol. 13, no. 7, 2003.

[3] M. Rahmani, R. Steffen, K. Tappayuthpijarn, G. Giordano, R. Bogenberger, E. Steinbach, "Performance Analysis of Different Network Technologies for In-Vehicle Audio and Video Communication", proceedings of the fourth international workshop on QoS in multiservice IP networks (QoS-IP 2008), Venezia, Italy, 2008.

[4] L. Pantel, L. C. Wolf, "On the impact of delay on real-time multiplayer games", proceedings of the $12^{\text {th }}$ international workshop on network and operating systems support for digital audio and video, New York, NY, USA, 2002.

[5] M. Rahmani, J. Hillebrand, W. Hintermaier, R. Bogenberger, E. Steinbach, "A Novel Network Architecture for In-Vehicle Audio and Video Streams", proceedings of the $2^{\text {nd }}$ IEEE/IFIP international workshop on broadband convergence networks (BcN 2007), Munich, Germany, 2007.

[6] S. Blake, D. Black, M. Carlson, E. Davies, Z. Wang, W. Weiss, "An Architecture for Differentiated Services", RFC 2475, IETF, 1998.

[7] Institute of Electrical and Electronics Engineers, "Information technology- Telecommunications and information exchange between systems- Local and metropolitan area networks- Specific Requirements- Part 3: Media Access Control (MAC) Bridges", ANSI/IEEE Std 802.1D, 1998

[8] ARINC Specification 664, "Aircraft Data Network, Part 7: Avionics Full Duplex Switched Ethernet (AFDX) Network", 2004. 